\title{
Le profil épidémiologique et biologique du cancer du sein à Fès-Boulemane (Maroc)
}

\author{
F. Abbass, ${ }^{1}$ S. Bennis, ${ }^{2}$ K. Znati, ${ }^{3}$ Y. Akasbi, ${ }^{4}$ J.K. Amrani, ${ }^{1}$ O. El Mesbahi ${ }^{4}$ et A. Amarti ${ }^{3}$
}

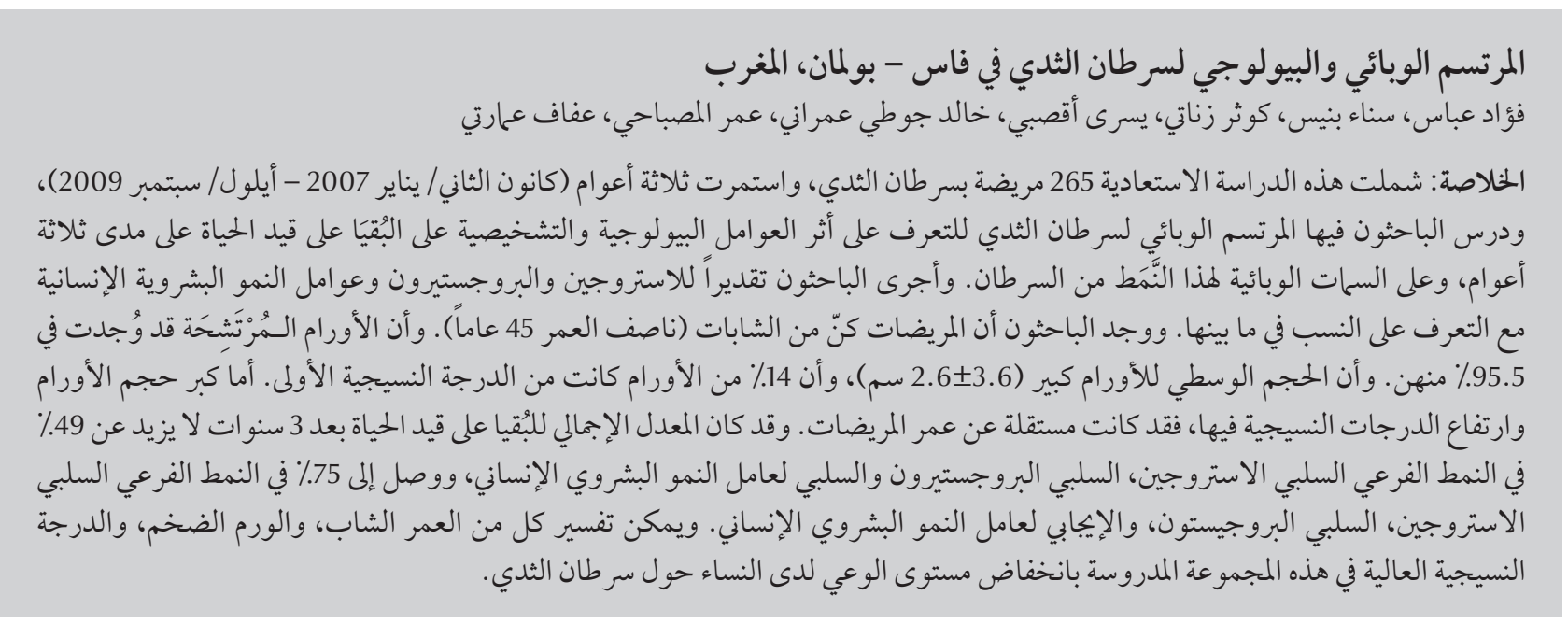

RÉSUMÉ Dans une étude rétrospective de 265 patientes atteintes d'un cancer du sein étalée sur trois ans (janvier 2007-septembre 2009), nous avons examiné le profil épidémiologique du cancer du sein afin de déterminer l'impact des facteurs pronostiques et biologiques des tumeurs sur la survie globale sur une période de trois ans, et sur l'épidémiologie de ce type de cancer. Les récepteurs œstrogène (RE), progestérone (RP) et facteurs de croissance épidermiques humains (HER2) ont été évalués et le statut RE/RP/HER2 a été déterminé. La population étudiée est jeune (âge médian 45 ans). Les tumeurs infiltrantes sont présentes chez 95,5\% des femmes. La taille moyenne des tumeurs est élevée $(3,6[$ ET 2,6] cm) et la proportion du grade histologique I est faible (14\%). La taille tumorale est importante et le grade histologique est élevé quelle que soit la tranche d'âge des patientes. Le taux de survie globale à trois ans n'est que de $49 \%$ pour le sous-type RE-/RP-/HER2- et de $75 \%$ pour le sous-type RE-/ RP-/HER2+, alors qu'il est de $96 \%$ pour le sous-type RE+/RP+/HER2-. L'âge jeune, la taille tumorale importante et le grade histologique élevé qui caractérisent notre population s'expliquent par l'absence de sensibilisation au cancer du sein.

\section{Epidemiological and biologic profile of breast cancer in Fez-Boulemane, Morocco}

In a retrospective study of 265 patients with breast cancer over 3 years (January 2007-September 2009) we examined the epidemiological profile of breast cancer to determine the impact of biological and prognostic factors on survival over 3 years and on the epidemiology of this cancer. Estrogen (RE), progesterone (RP) and human epidermal growth factor receptors (HER2) were evaluated and RE/RP/HER2 status determined. The patients were young (median age 45 years). Invasive tumours were found in 95.5\% of the women. The average tumour size was big [3.6 (SD 2.6) $\mathrm{cm}$ ] and only $14 \%$ were histological grade I. Large tumour size and high histological grade were independent of patient's age. Overall survival at 3 years was only 49\% for the RE-/RP-/HER2- subtype and 75\% for the RE-/RP-/HER2+ subtype, while it was $96 \%$ for the RE+/RP+/HER2- subtype. The young age, large tumour size and high histological grade in our population suggest a lack of awareness of women about breast cancer.

${ }^{7}$ Faculté des Sciences et Techniques de Fès, Fès (Maroc).

${ }^{2}$ Faculté de Médecine et de Pharmacie ; ${ }^{3}$ Service d'Anatomie pathologique ; ${ }^{4}$ Service d'Oncologie médicale, CHU Hassan I/ de Fès, Fès (Maroc) (Correspondance à adresserà S.Bennis : sabennis@yahoo.fr).

Reçu : 29/09/10; accepté : 27/03/11 


\section{Introduction}

Le cancer du sein est la première cause de mortalité chez la femme en Europe. C'est un cancer qui touche plus de 37000 nouveaux cas par an en France et est responsable de plus de 11000 décès estimés en 2000 [1]. Il est également considéré comme étant le premier cancer de la femme au Maroc avec la particularité d'affecter fréquemment la femme jeune (âge médian de 45 ans), comme cela a été rapporté en Jordanie [2,3]. Ainsi, le Maroc rejoint la plupart des pays occidentaux puisque la fréquence du cancer du sein est en augmentation régulière depuis la dernière décennie, et représente un problème majeur de santé publique. L'âge jeune moins de 40 ans - est considéré comme l'un des principaux facteurs de mauvais pronostic [1], mais il y a également la taille de la tumeur, le grade histologique de Scarff, Bloom et Richardson (SBR) etl'envahissement ganglionnaire qui sont des facteurs pronostiques péjoratifs et qui orientent la prise en charge thérapeutique. La détermination et l'exploration d'autres facteurs pronostiques et prédictifs de la réponse aux traitements basés sur l'expression des récepteurs des œstrogènes, de la progestérone et de la protéine HER2/neu (statut RE/ $\mathrm{RP} / \mathrm{HER} 2$ ) favorisent une sélection des patientes en vu du meilleur choix thérapeutique. Les tumeurs positives aux récepteurs hormonaux sont susceptibles de répondre à l'hormonothérapie et cette réponse est associée à un bon pronostic [47], alors que l'amplification du gène HER2 dans les tumeurs invasives rend le pronostic mauvais avec une augmentation des récidives et une survie diminuée, comme cela a été rapporté dans la littérature [4-8]. Donc la détermination des sous-types biologiques basés sur le statut RE/RP/ HER2 serait incontournable puisque ceux-ci s'associent à la durée de survie et au développement des métastases [9]. Par ailleurs, il a été prouvé que le diagnostic du cancer du sein à un stade précoce du développement assurerait une prise en charge thérapeutique beaucoup plus efficace [10] et moins lourde [11] et permettrait ainsi une amélioration du pronostic global. Grosclaude et al. [12] estimaient la survie à cinq ans à $88 \%$ pour un stade initial T1 (taille tumorale), 74 \% à T2, $62 \%$ à T3 et $44 \%$ à T4 [1]. Cependant, cette estimation de survie est réduite en présence d'un grade histologique élevé ou d'un envahissement ganglionnaire [9].

L'objectif de cette étude, réalisée pour la première fois au Maroc, était d'évaluer le profil épidémiologique du cancer du sein dans la région de Fès à travers les patientes recrutées dans le Centre hospitalier universitaire (CHU), puis de déterminer les facteurs cliniques, biologiques et pathologiques qui conditionnaient la survie globale et enfin de montrer l'intérêt de la mise en place d'un programme de dépistage organisé.

\section{Méthodes}

C'est une étude rétrospective réalisée sur 265 patientes de la région Nord-Est du Maroc, âgées de 18 à 80 ans et colligés dans le CHU entre janvier 2007 et septembre 2009. Chaque tumeur a été analysée et gradée par un pathologiste du CHU selon le système de Scarff, Bloom et Richardson (SBR) $[13,14]$ puis une étude immunohistochimique (IHC) a été réalisée pour la classification biologique des tumeurs invasives.

\section{Étude immunohistochimique (IHC)}

Des coupes de $4 \mu \mathrm{m}$ sont traitées et incubées avec l'anticorps (RE : clone ER1D5; RP : clone PR10A9 [Immunotech]) selon le protocole de Dako. L'expression des récepteurs hormonaux est évaluée en pourcentage du marquage nucléaire des cellules tumorales et un pourcentage de plus de $10 \%$ est considéré comme positif. L'expression du HER2/neu est évaluée selon le protocole du kit HercepTest ${ }^{\circ}$ (Dako) et les résultats sont exprimés en score selon les recommandations de l'American Society of Clinical Oncology (ASCO) [15]. Les scores 2 sont validés positifs ou négatifs par la technique d'hybridation in situ par fluorescence (FISH).

\section{Hybridation fluorescente in situ (FISH)}

Le protocole Vysis avec le kit Path Vysion HER-2 DNA Probe (VysisAbbott) est utilisé dans l'évaluation de l'expression du gène HER2 qui est considéré amplifié si le rapport des signaux de LSI-HER2/CEP-17 est supérieur à 2 .

\section{Analyse statistique}

Les données issues des dossiers cliniques et des comptes rendus anatomo-pathologiques sont recueillies, puis codées et saisies sur Microsoft Office Excel 2003. L'analyse est réalisée au moyen du logiciel Epi Info version 3.4 sur tous les cancers invasifs.

Les différentes variables sont examinées à l'aide du test $\chi^{2}$ ou du test Fisher. Le taux de survie globale (OS) a été évalué par la méthode de KaplanMeier, et le test log-rank. $(p \leq 0,05)$ est une valeur statistiquement significative.

\section{Résultats}

L'étude a porté sur 265 patientes recrutées entre janvier 2007 et septembre 2009. L'analyse histologique a révélé que 4,5\% des tumeurs (soit 12 patientes) montrent un carcinome in situ (CIS) et 95,5\% (soit 254 patientes) présentent un carcinome mammaire invasif. L'analyse qui suit 
a intéressé uniquement les tumeurs invasives, soit 254 patientes, dont toutes les variables sont notées (âge, taille tumorale, grade histologique et statut RE/RP/HER2).

Cette population provenant de la région Nord-Est du Maroc est caractérisée par un âge jeune, avec un âge médian de 45 ans, et des extrêmes allant de 18 à 80 ans. En outre, $17 \%$ ont moins de 35 ans et $75 \%$ moins de 55 ans.

L'étude histologique a montré que le type histologique prédominant des tumeurs était le carcinome canalaire infiltrant (CCI) dans 87,8 \% des cas, suivi du carcinome lobulaire infiltrant (CLI 4,7\%), puis le carcinome métaplasique $(2,4 \%)$ et le carcinome médullaire (2\%). Les autres types histologiques étaient plus rares.

La taille tumorale moyenne est de 3,6 (ET 2,6) cm, avec $30 \%$ des tumeurs ayant une taille très importante $\ll \mathrm{T} 3 »$. La proportion des grades histologiques II et III est élevée : elle est de 56,1\% et $39,4 \%$ respectivement, alors que la proportion du grade histologique I est faible, estimée à $14 \%$.

L'étude immunohistochimique a révélé que $72 \%$ des tumeurs sont positives aux récepteurs hormonaux avec $54,7 \%$ positives aux RE, $66 \%$ positives aux RP; $26 \%$ des tumeurs surexpriment la protéine HER2 (score 3 ), et $8 \%$ ont une expression douteuse (score 2). Ces derniers ( $8 \%$ ) sont validés par cytogénétique moléculaire (FISH) et $25 \%$ de ces tumeurs se sont révélées positives.

L'expression des RE augmente avec l'âge, passant de $50 \%$ chez les patientes âgées de 18-40 ans à $65 \%$ chez les patientes âgées de 61-80 ans. Cependant, l'expression des RP reste relativement stable quelle que soit la tranche d'âge des patientes (65\%). La surexpression de la protéine HER2 est estimée à $30 \%$ jusqu'à l'âge de 60 ans, puis elle baisse à $21 \%$ pour la tranche d'âge de 61-80 ans (Figure 1).
La figure 1 montre que, quel que soitl'âge, la proportion des grades SBR II et III est élevée, particulièrement pour les patientes de 18-40 ans chez lesquelles le pourcentage est estimé à $56 \%$ pour SBR II et $29 \%$ pour SBR III. La proportion des tumeurs grade SBR I augmente avec l'âge; en revanche l'inverse se produit avec les tumeurs grade SBR III.

Dans cette étude, la taille tumorale est corrélée significativement avec le grade $\operatorname{SBR}(p=0,012) ; 47 \%$ des tumeurs $\mathrm{T} 1$ sont de grade SBR I, $32 \%$ des tumeurs T3 sont de grade SBR II et $33 \%$ des T3 sont de grade SBR III (Figure 2). Toutefois, l'expression des RE baisse significativement avec les grades SBR II et III $(p=0,0002)$.

La recherche par immunohistochimie du statut RE/RP/HER2 dans les tumeurs a permis de distinguer huit sous-types biologiques, que nous avons classés selon l'ordre de la meilleure durée de survie comme cela

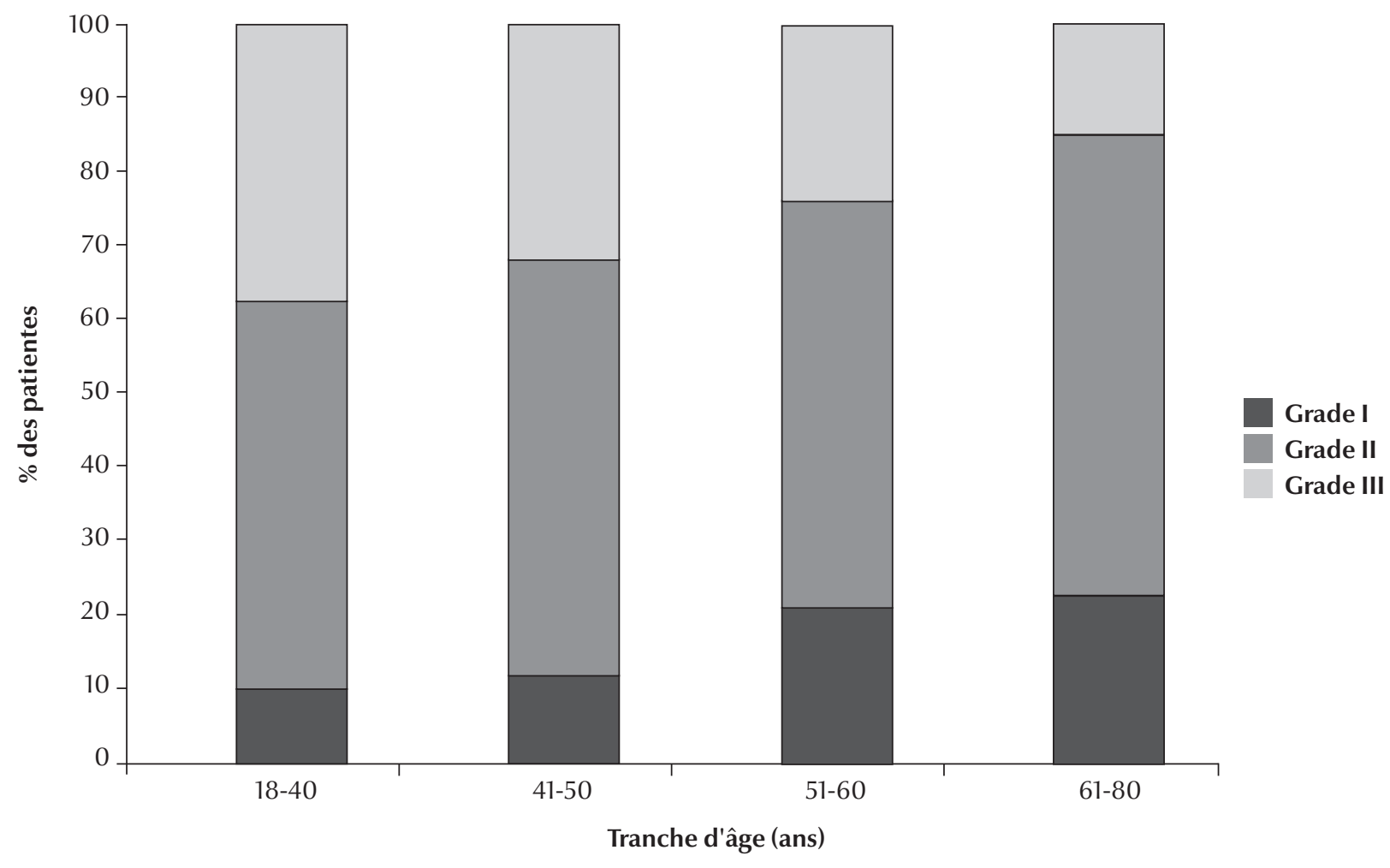

Figure 1 Détermination du grade histologique en fonction de l'âge des patientes 


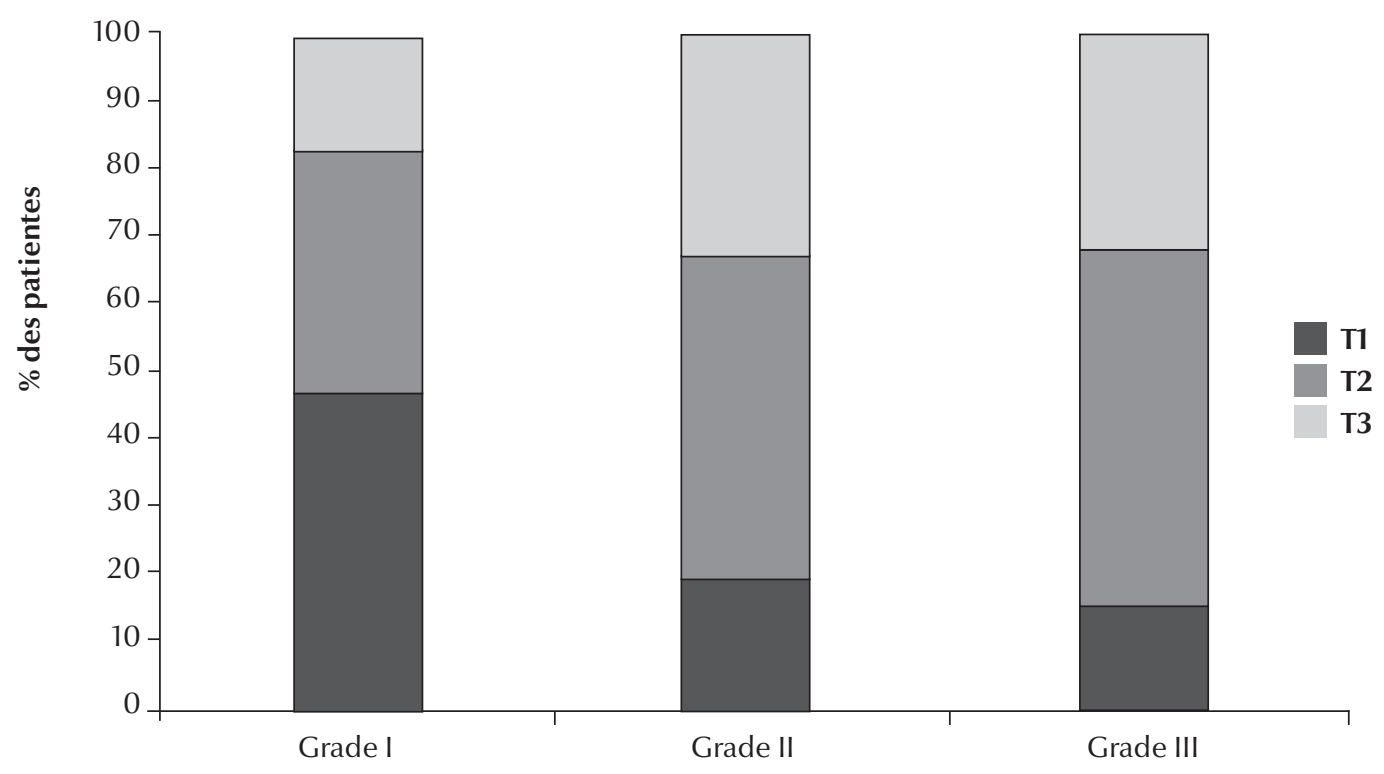

Figure 2 Corrélation du grade Scarff, Bloom et Richardson (SBR) avec la taille tumorale

a été décrit précédemment [16]: RE+/ $\mathrm{RP}+$ /HER2-, RE+/RP-/HER2-, RE+/ $\mathrm{RP}+/ \mathrm{HER} 2+$, RE+/RP-/HER2+, RE-/ $\mathrm{RP}+/ \mathrm{HER} 2-, \mathrm{RE}-/ \mathrm{RP}+/ \mathrm{HER} 2+, \mathrm{RE}-/$ RP-/HER2-, RE-/RP-/HER2+.

Le sous-type RE+/RP+/HER2représente la majorité des cas $(38,6 \%)$ suivi du type RE-/RP-/HER2- avec $19,3 \%$ alors que les sous-types RE+/ RP-/HER2+, RE+/RP-/HER2- et $\mathrm{RE}-/ \mathrm{RP}+/ \mathrm{HER} 2+$ sont faiblement représentés $(1,2 \%, 4,3 \%$ et $6,3 \%$ respectivement), ne permettant pas une analyse statistiquement fiable et donc, pour une clarté des résultats, ces soustypes biologiques ne figurent pas par la suite.

Dans cette étude, c'est l'âge jeune et la taille tumorale importante qui prédominent dans tous les sous-types biologiques. La classification de ces sous-types biologiques en fonction des tranches d'âge montre toujours une prédominance du sous-type RE+/ RP+/HER2-, suivi du sous-type RE-/ RP-/HER2-. Les sous-types RE-/RP-/ HER 2+ et RE-/RP+/HER2- sont représentés dans une proportion assez importante, particulièrement pour un âge de moins de 50 ans (Figure 3). La proportion du sous-type $\mathrm{RE}+\mathrm{RP}+$ / HER+ est de 5 à $10 \%$ quelle que soit la tranche d'âge. Si on corrèle les sous-types biologiques $\mathrm{RE}+/ \mathrm{RP}+/$ HER2-, RE-/RP-/HER2-, RE-/RP-/ HER2+ avec la taille tumorale et le grade histologique SBR, on remarque que le sous-type RE+/RP+/HER2est caractérisé par une proportion élevée de T2 $(48 \%)$ et de grade SBR I (23\%) ; par contre le soustype RE-/RP-/HER2- montre une proportion élevée de T3 (44\%) et de grade SBR III (33\%). Le sous-type RE-/RP-/HER2+ montre plutôt une proportion élevée de T2 (60\%) et de grade SBR II (39\%). Les tumeurs de grade SBR II sont largement représentées dans les trois sous-types biologiques (plus de $48 \%$ ). L'étude statistique des relations entre les trois facteurs (l'âge médian, la taille moyenne des tumeurs, le grade SBR, l'expression des protéines RE/RP/ HER2) montre que, quel que soit le sous-type biologique, les patientes sont jeunes (âge médian inférieur ou égal à 51 ans), les tumeurs ont une taille moyenne supérieure à $2,6 \mathrm{~cm}$ et les grades SBR II et III sont importants (Tableau 1).
La détermination de la survie globale des patientes à trois ans montre qu'elle est très faible pour le sous-type RE-/RP-/ HER2- (49\%), suivi du sous type RE-/ RP+/HER2- (53\%) puis RE-/RP-/ HER2+ $(75 \%)$. En revanche, cette survie est significativement élevée pour les sous- types RE+/RP+/HER2- et RE+/ $\mathrm{RP}+/ \mathrm{HER} 2+$, estimée à $96 \%$ et $92 \%$ respectivement (test log-rank, $\mathrm{p}=0,035$ ).

\section{Discussion}

Les critères pronostiques clinicopathologiques et biologiques sont reconnus depuis longtemps comme indispensables à la prise en charge du cancer du sein. L'expression des récepteurs hormonaux est un facteur prédictif de ce cancer $[17,18]$ et les tumeurs montrant une surexpression de la protéine HER2 sont de mauvais pronostic; donc la distinction des différents sous-types biologiques basés sur le statut RE/RP/HER2 et l'établissement des caractéristiques biologiques et clinico-pathologiques sont nécessaires pour assurer une meilleure prise en charge des patientes. En outre, une étude américaine réalisée 


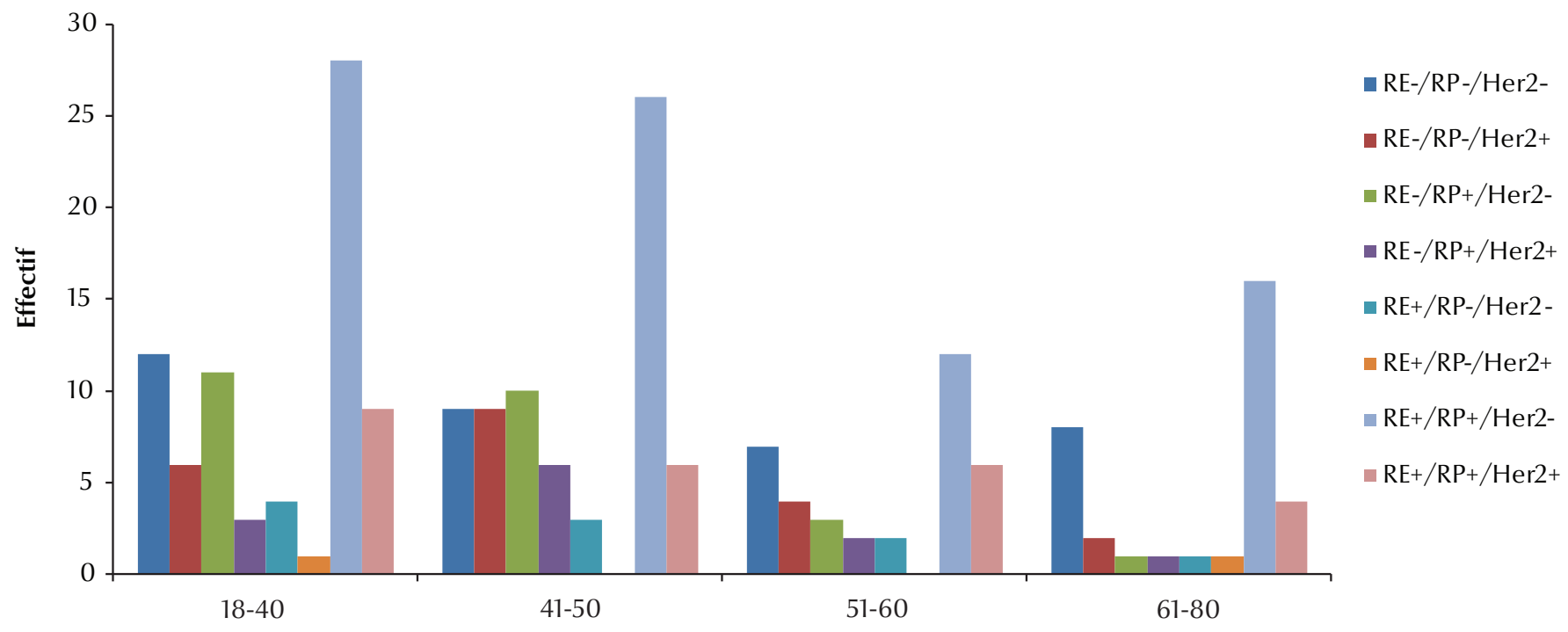

Tranche d'âge (ans)

Figure 3 Distribution des sous-types biologiques en fonction de l'âge (RE : récepteurs des oestrogènes ; RP : récepteur de la progestérone ; HER2 : récepteur pour les facteurs de croissance épidermique humains ; SBR : Scarff, Bloom et Richardson)

en 2009 sur quatre ethnies différentes a montré que le statut RE/RP/ HER2, l'âge et l'origine des patientes influencent le pronostic du cancer du sein [19].

L'objectif de cette étude rétrospective étalée sur une période de 3 ans (janvier 2007 septembre 2009), réalisée pour la première fois au Maroc sur des patientes atteintes de cancer du sein et colligées dans le centre hospitalier universitaire de Fès, était d'abord d'évaluer le profil épidémiologique du cancer du sein dans cette région, puis de déterminer les facteurs pronostiques caractéristiques de ces tumeurs.

Après analyse histologique des différentes tumeurs, nous avons constaté qu'il y a une faible proportion de carcinomes in situ (4,5\%), alors que les tumeurs infiltrantes sont les plus prépondérantes (95,5\%) dont $87,8 \%$ sont des CCI, $4,7 \%$ des cas correspondent à des CLI puis 2,4\% sont des carcinomes métaplasiques et $2 \%$ sont des carcinomes médullaires. L'âge médian des patientes atteintes de ce cancer est particulièrement bas (45 ans), avec $17 \%$ des cas ayant moins de 35 ans et $75 \%$ ont moins de 55 ans ; c'est un âge jeune, comme cela a été décrit pour la population jordanienne $[3,20]$. En revanche, le cancer du sein en Occident survient à un âge plus avancé (âge médian de 55 ans) et seulement $3 \%$ des patientes ont moins de 35 ans [21].

La taille moyenne des tumeurs est élevée, supérieure à 3,6 (ET 2,6) cm, valeur également similaire à celle retrouvée en Jordanie, mais très différente de celle observée dans les études européennes [21] et américaines [19] où la taille moyenne est inférieure à $2 \mathrm{~cm}$. Par ailleurs, le pourcentage des tumeurs de grade I

\begin{tabular}{lccc}
\hline \multicolumn{2}{c}{ Tableau 1 Caractéristiques des différents sous-types biologiques : fréquence, taille moyenne et âge médian } \\
\hline Statut RE/RP/HER2 & $\%$ & Age médian (ans) & Taille moyenne $(\mathbf{c m})$ \\
RE+RP+HER2- & 38,6 & 45 & 3,2 \\
RE+RP-HER2- & 4,3 & 45 & 5,9 \\
RE+RP+HER2+ & 10,6 & 44 & 3,2 \\
RE+RP-HER2+ & 1,2 & 51 & 3,0 \\
RE-RP+HER2- & 10,2 & 42 & 4,6 \\
RE-RP+HER2+ & 6,3 & 44 & 4,3 \\
RE-RP-HER2- & 19,3 & 47 & 4,0 \\
RE-RP-HER2+ & 9,4 & 45 & 2,6 \\
\hline
\end{tabular}

$R E$ : récepteurs des oestrogènes; $R P$ : récepteur de la progestérone; HER2 : récepteur pour les facteurs de croissance épidermique humains. 
au diagnostic est très faible ( $14 \%$ au lieu de $30 \%$ en Europe), alors que les grades III sont du même ordre de grandeur (30,5\% versus $30 \%)$. Les tumeurs de grade II sont les plus fréquentes, mais de façon plus marquée au Maroc (55,5\%) qu'en Europe (42\%) [21]. Nous avons également constaté que les proportions des grades SBR II et III sont élevées quelle que soit la tranche d'âge ( $56 \%$ et $29 \%$ ) et ces grades histologiques élevés sont corrélés, d'une part, avec les tailles tumorales importantes (T2 et T3) et d'autre part, avec une baisse d'expression des RE. L'importance des facteurs de mauvais pronostic, retrouvée dans les tumeurs des patientes de la région de Fès, ne peut s'expliquer que par le diagnostic tardif dû à l'absence d’un programme de dépistage.

L'étude IHC basée sur le statut $\mathrm{RE} / \mathrm{RP} / \mathrm{HER} 2$ révèle la présence de huit sous-types biologiques avec la prévalence du sous-type $\mathrm{RE}+\mathrm{RP}+$ / HER2-(38,6\%), suivi du sous-type RE-/RP-/HER2- avec 19,3\% des cas. Ce sous-type triple négatif (RE-/RP-/ HER2-) est présent à un pourcentage élevé chez les femmes âgées de moins de 40 ans $(33,3 \%)$, comme cela a été trouvé dans la population afroaméricaine [19].

Dans cette étude, quel que soit le sous-type biologique, c'est l'âge jeune, la taille tumorale moyenne importante (allant de 2,6 à 5,9 cm) et le grade histologique SBR II et III qui prédominent. Cela encore s'explique par l'absence de sensibilisation des femmes (particulièrement les femmes du milieu rural) et l'absence d'une stratégie nationale de dépistage.

Après détermination de la survie globale à trois ans, nous avons montré que malgré la taille importante des tumeurs, le grade histologique avancé, nos résultats sont concordants avec la littérature [19] : les sous-types RE+/ $\mathrm{RP}+/ \mathrm{HER} 2+$ et RE+/RP+/HER2sont de bons pronostics, alors que le sous-type triple négatif est très agressif et le sous-type RE-/RP+/HER2- est d'agressivité modérée. Cependant, notre analyse est limitée vu qu'on s'est basé sur un recul de trois ans.

À travers cette étude, nous avons pu constater que le Maroc est en retard de quelques années par rapport à l'Europe et aux États-Unis. En effet, dans ces pays industrialisés où le dépistage organisé est en place depuis 20 à
30 ans, celui-ci a contribué à un diagnostic précoce des cancers infiltrants (petite taille tumorale, faible grade histologique et absence d'envahissement ganglionnaire) $[1,22,23]$, puis à une augmentation de l'incidence des cancers in situ. Cela a pour conséquence l'amélioration de la survie et la baisse de morbidité des patientes atteintes de cancer du sein. Dans notre contexte, il semble que la mise en place d'un programme de dépistage au Maroc, particulièrement dans la région de Fès, est une nécessité. Par ailleurs, notre population est très jeune ( $75 \%$ ont moins de 50 ans) et donc l'établissement d'un programme de dépistage serait plus efficace si celuici est instauré à lâge de 40 ans au lieu de 50 ans. D'autres études dans ce sens ne peuvent qu'apporter une réponse et permettre des analyses comparatives à nos résultats.

\section{Remerciements}

Nous remercions les Dr Laila CHBANI et Hinde ELFATEMI pour leur contribution à la lecture histologique des lames.

\section{Références}

1. Molinié F et al. Incidence et facteurs pronostiques des cancers du sein découverts au cours et en dehors du programme de dépistage organisé en Loire-Atlantique (1991-2002) [Incidence and prognostic factors of breast cancer diagnosed within and outside the organised screening program in the department of Loire-Atlantique (1991-2002)]. Revue d'Épidémiologie et de Santé Publique, 2008, 56:41-49.

2. Dajani YF, Al-Jitawi SA. A study of 405 breast tumors in Jordanians using the Revised WHO classification. Tropical and Geographical Medicine, 1987, 39:182-186.

3. Abalkail AA et al. The role of young population structure in determining age distribution of breast cancer in Jordan. Journal of the Bahrain Medical Society, 2003, 15:28-33.

4. Lamy PJ et al. Classification moléculaire des cancers du sein : utilité en clinique [Molecular classification of breast cancers: clinical utility]. Médecine nucléaire, 2010, 34(1):32-43.

5. Osborne CK et al. The value of estrogen and progesterone receptors in the treatment of breast cancer. Cancer, 1980, 46(Suppl.):2884-2888.
6. Clark GM, McGuire WL. Steroid receptors and other prognostic factors in primary breast cancer. Seminars in Oncology, 1988, 15(Suppl. 1):20-25.

7. Early Breast Cancer Trialists' Collaborative Group. Systemic treatment of early breast cancer by hormonal, cytotoxic, or immune therapy. 133 randomised trials involving 31,000 recurrences and 24,000 deaths among 75,000 women. Early Breast Cancer Trialists' Collaborative Group. Lancet, 1992, 339:71-85.

8. Ross JS, Gray GS. Targeted therapy for cancer: the HER-2/neu and Herceptin story. Clinical Leadership \& Management Review, 2003, 17:333-340.

9. Belkacémi Y et al. Intérêt des classifications moléculaires pour prédire la rechute locale et la diffusion métastatique des cancers du sein [The use of breast cancer subtype classification to predict local and distant recurrence. A review]. Cancer Radiothérapie, 2008, 12:2577-2583.

10. Engel $\mathrm{J}$ et al. The process of metastasisation for breast cancer. European Journal of Cancer (Oxford, England), 2003, 39:17941806.

11. Fédération nationale des Centres de Lutte contre le Cancer (FNCLCC). Comprendre le cancer du sein. Guide d'information 
et de dialogue à l'usage des patientes et de leurs proches, mise à jour 2002 [Understanding breast cancer. An information and dialogue guide for patients and their families, 2002 update]. Paris, Fédération nationale des Centres de Lutte contre le Cancer, Collection SOR SAVOIR PATIENT (Standards, Options et Recommandations pour le Savoir des Patients), 2002:1-160.

12. Grosclaude $\mathbf{P}$ et al. Survival of women with breast cancer in France: variation with age, stage and treatment. Breast Cancer Research and Treatment, 2001, 70:137-143.

13. Tavassoli FA, Devilee P, eds. Pathology and genetics of tumours of the breast and female genital organs. Lyon, IARC Press, 2003 (World Health Organization classification of tumours, No. 4).

14. Elston CW, Ellis IO. Pathological prognostic factors in breast cancer. I. The value of histological grade in breast cancer: experience from a large study with long-term follow-up. Histopathology, 1991, 19:403-410.

15. Wolff AC et al. American Society of Clinical Oncology; College of American Pathologists. American Society of Clinical Oncology/College of American Pathologists guideline recommendations for human epidermal growth factor receptor 2 testing in breast cancer. Journal of Clinical Oncology, 2007, 25:118-145.

16. Parise $\mathrm{C}$ et al. Breast cancer subtypes as defined by the estrogen receptor (ER), progesterone (RP), and the human epidermal growth factor receptor2 (Her2) among women with invasive breast cancer subtypes in California 1999-2004. Breast Journal, 2009, 15:593-602.

17. Grann VR et al. Hormone receptor status and survival in a population-based cohort of patients with breast carcinoma. Cancer, 2005, 103:2241-2251.

18. Dunnwald LK, Rossing MA, Li Cl. Hormone receptor status, tumor characteristics, and prognosis: a prospective cohort of breast cancer patients. Breast Cancer Research, 2007, 9:R6.

19. Parise C, Bauer K, Caggiano V. Variation in breast cancer subtypes with age and race/ethnicity. Critical Reviews in Oncology/ Hematology, 2010, 76(1):44-52.

20. Almasri NM, Al Hamad M. Immunohistochemical evaluation of human epidermal growth factor receptor 2 and estrogen and progesterone receptors in breast carcinoma in Jordan. Breast Cancer Research, 2005, 7:R598-R604.

21. Blamey RW et al. ONCOPOOL - a European database for 16,944 cases of breast cancer. European Journal of Cancer, 2010, 46:56-71.

22. Ernst MF et al. Breast cancers found by screening: earlier detection, lower malignant potential or both? Breast Cancer Research and Treatment, 2002, 76:19-25.

23. Gill PG et al. Detection by screening mammography is a powerful independent predictor of survival in women diagnosed with breast cancer. Breast, 2004, 13:15-22.

\section{Les femmes et la santé}

Le présent rapport, Les femmes et la santé, utilise les données actuellement disponibles pour faire le bilan de la santé des filles et des femmes dans le monde entier et pour attirer lattention sur les conséquences à attendre et ce qu'il en coûte si les problèmes de santé des femmes ne sont pas pris en charge au bon moment. Il montre que bien que la santé des filles et des femmes se soit beaucoup améliorée au cours des 60 dernières années, les progrès sont très inégalement répartis. Dans de nombreuses parties du monde, la vie des femmes - de lenfance à la vieillesse - est amputée par les maladies évitables et les décès prématurés. Le rapport met en lumière les points communs entre les problèmes de santé auxquels doivent faire face les femmes dans le monde entier mais attire aussi l'attention sur les différences qui découlent de modes de vie très variés.

Le rapport donne une idée des mesures à prendre pour améliorer la santé des filles et des femmes et il a pour but de livrer les informations nécessaires au dialogue politique et d'encourager les pays, les institutions et les partenaires du développement à agir. Il met en particulier laccent sur le besoin urgent d'un leadership politique et institutionnel plus cohérent et sur la nécessité de parler davantage de la santé des femmes et de mobiliser des ressources suffisantes pour sauver plus de vies et améliorer la santé des filles et des femmes. Comme lindique de rapport, soccuper de la santé des femmes est une approche nécessaire et utile pour renforcer les systèmes de santé d’une façon générale, dans lintérêt de tous. 